\title{
The lived experience of a 24-hour support center for persons with psychiatric disabilities: Making me feel almost like an ordinary person
}

\author{
Ann-Christin Janlöv ${ }^{1}$, Irené Persson ${ }^{1}$, Agneta Berg ${ }^{1,2}$ \\ ${ }^{1}$ School of Health and Society, Kristianstad University, Kristianstad, Sweden \\ ${ }^{2}$ Department of Nursing, Health and Culture, University West, Trollhättan, Sweden \\ Email: ann-christin.janlov@hkr.se
}

Received 23 October 2013; revised 19 December 2013; accepted 5 January 2014

Copyright (c) 2014 Ann-Christin Janlöv et al. This is an open access article distributed under the Creative Commons Attribution License, which permits unrestricted use, distribution, and reproduction in any medium, provided the original work is properly cited. In accordance of the Creative Commons Attribution License all Copyrights (C) 2014 are reserved for SCIRP and the owner of the intellectual property Ann-Christin Janlöv et al. All Copyright (C) 2014 are guarded by law and by SCIRP as a guardian.

\section{ABSTRACT}

This study illuminates nine psychiatric disabled persons' lived experience of a newly established community-based service center open around the clock. This new 24-hour support center (SC) was established in 2011 in a Swedish community to better match and facilitate the disabled persons' needs. In order to illuminate the disabled person's experiences individual interview was performed. A phenomenological-hermeneutical method inspired by Paul Ricoeur was used to interpret the texts. After a naive reading, a structural analysis revealed two themes: 1), becoming aware of myself as a person, and 2) having a lifeline and belongingness. The comprehensive understanding was interpreted as meaning "Making me feel almost like an ordinary person", which incorporated the person's past with their present together with a direction for the future and hope for a more fulfilling life.

\section{KEYWORDS}

Psychiatric Disability; Lived Experience; Community Mental Health Service; 24-Hour Service Center; Phenomenological-Hermeneutical Method; Mental Health Nursing

\section{INTRODUCTION}

Adequate support such as community-based service centers open around the clock may be crucial for the wellbeing of people with psychiatric disabilities living in the community. This is a vulnerable group of persons regardless of where they live [1]. In Sweden a recent follow-up report [2] highlighted the situation for these people; alarming differences still exist in comparison to the general population regarding health, death, care and services, and living circumstances. Nationally the need for support facilities better matched to disabled persons' individual needs has been emphasized, as having the need to facilitate their participation in community life [3]. In order to provide greater stability in the lives of people with psychiatric disabilities, a new 24-hour-a-day support center (SC) was established in a community in the southeast of Sweden. The start of the SC was based on psychiatrically disabled persons' own desires for such a place. They took part, together with the staff, in the design. Because this was a new service it seemed important to explore what the SC has meant to both users and nurses. Interviews with the nurses showed that they experienced the SC supportespecially the night-time availability—making a positive difference to the users [4].

The ability of people with psychiatric disabilities to get the care and services they need is a prerequisite to stability in their daily lives. However, how to organize attractive support for this sensitive group can be a puzzle. According to Rosenberg [5], people with psychiatric illness can be aware of their need for support, but refrain from seeking it for fear of falling under the control of others. This can be seen as a sort of withdrawal. An Australian study showed that the negative effects of and the pain of social exclusion lasted longer in individuals with schizophrenia, compared with non-clinical controls [6]. Withdrawal can be a strategy to prevent pain. Lundberg [7] studied experiences of stigmatization and found that of 200 participants with psychiatric illness, half of them sometimes, often, or very often avoided people because they experienced being devalued. Such social withdrawal 
can be a result of the psychiatric disabilities but also a consequence of stigmatization and/or feeling stigmatized, or both, creating a double exposure, which may worsen their difficulties and their tendencies toward isolation [8]. A vicious circle like this must be broken because it can counteract recovery and stop the transition from feelings of marginalization and abnormality to feelings of acceptance as an ordinary person among others in the community.

The life situations of persons with serious psychiatric illness in Sweden have been found in previous studies to mean exposure to risks such as homelessness, drug addiction, comorbidity, violence and crime, somatic illness, and lifestyle problems [9]. In short, they are described in a report [2] as being at high risk for having poor living circumstances, ill health, and premature death. Swedish research has shown how persons with psychiatric disabilities themselves experience their daily life in the community. Nyström [10] found that the various forms of care and services constituted an important part of these people's daily lives. Meaningful relationships without therapeutic goals were an expressed need. Being accepted even with their problems as the persons they were, without demands that they change, meant being able to live a good life. Researchers [11] asked people with long-term mental illness to take photos of matters of importance to them and reflect on them. Varying views of life revealed key existential issues concerning thoughts about God and life and death; the meaning of relationships with others; animals giving meaning to their life without making demands; and the symbolic bearing of objects on life. These were issues they otherwise never discussed. Other findings [12] described certain psychosocial disadvantages among this group of people. Contradictory thoughts, feelings, and actions were reflected by their knowledge of what to do and at the same time their lack of initiative; they felt lonely but were unable to establish friendships. Klamas [13] found people with psychiatric disabilities considered relational qualities to be a significant feature for how they experienced support. A variety of support sources such as family, friends, and co-workers as well as care and service staff stood out as a prerequisite to developing social bonds and roles within the community.

Psychiatric health care and social services in Sweden are rather complex because the responsibility is shared by two systems: 1) the county councils and 2) the communities and two pieces of legislation. The Health and Medical Services Act [14] regulates the county councils, and the Social Services Act [15] regulates the communities. All health care and social services are mainly financed by taxes. While the county councils provide specialist care, emergency services, and inpatient and outpatient psychiatric health care, the primary care/health centers are responsible for all health and medical care and less serious psychiatric illness. More serious psychiatric illness is referred to specialist psychiatric health care. The communities have the legal responsibility to provide housing, activities, and needs-assessed support to people with psychiatric disabilities [15,16]. Functions like case management can be an integrated part of the organization. Day centers and meeting points at daytime are common. Regardless of their responsibility, the communities have had difficulties finding forms of adapted care and services to people with psychiatric disabilities. In order to improve care and services, action was taken to reduce relapse and the number of hospitalizations. National Guidelines [3] were commissioned to provide recommendations based on evidence. The guidelines stressed different types of support needed to achieve greater stability for the disabled people living in assisted as well as ordinary housing. The forms of care support and service support vary and are not sufficiently available and comprehensive in the 290 Swedish communities. Moreover, people can fall between organizational borders [2]. A report focusing on whether people with psychiatric disabilities got community support according to their needs showed deficiencies regarding housing support. They requested support available during flexible hours and at nights, support was extremely rare [17]. Users in England wanted the development of 24-hour community support, seven days a week, together with care for individual needs [18].

\section{Background of the Study}

In 2006 a collaboration between the county council and a community in southern Sweden, Region Skåne was started due to a user survey that had revealed psychiatric disabled persons' dissatisfaction with their care and social service. They wanted a place to turn to, to call, or to visit at any time, 24 hours a day. As a result, a new, nonmedical center was established at year end 2009 to provide service based on persons' desires for support. It operated around-the-clock and offered anonymity by not requiring registration or documentation. Because this new community service has been explored from the perspective of the nurses [4], the aim of this study was to illuminate the psychiatrically disabled lived experience of this newly established SC.

\section{METHOD}

A qualitative life-world approach was chosen to direct the design. It concerns the individual experience of being in the world-here in terms of having psychiatric disabilities. The life-world is central in both phenomenology and hermeneutic philosophies, which constitute a base in the phenomenological-hermeneutical method developed by Lindseth and Norberg [19]. This method is inspired 
by the theory of interpretation presented by Paul Ricoeur $[20,21]$ and was chosen so that interpretation of the interview texts could be made through uncovering the meaning of the lived experience of nine persons' narratives.

\subsection{Context}

The context of the study was a city of an average-sized community of 50,000 inhabitants in Skåne in southern Sweden. The local community psychiatric care and service organization also encompassed two smaller communities-altogether 70,000 inhabitants. The psychiatric care in the city comprised two general psychiatric care teams, a psychosis team, a hospital ward for general psychiatry, a ward for forensic psychiatric care, outpatient care, and day care. The emergency department was placed in a larger city 3 - 4 Swedish miles away. The community psychiatric health service provided two facilities with support and one meeting point with activities open daytimes Monday through Friday.

\subsection{Setting}

The SC was placed in a building in the city center, which also encompassed assisted housing apartments. The SC had a top floor that included two bedrooms called "support beds", which could be used for a maximum of 48 hours if needed. The staff consisted of 16 licensed psychiatric practical nurses (LPPN) 24 hours a day. A registered psychiatric health nurse headed the unit and was responsible for daily management. The persons using SC were between 20 and 70 years of age. Most of them had prior experiences with mental health services, but those without prior experience also used SC.

\subsection{Participants}

Of the approximately 50 persons visiting SC, eleven wellspoken persons were asked by the nurses if they were interested in participating in the study. All agreed to participate; but two of them reported being unable to participate at the day of the planned interview. Of the nine who participated, two were women and seven men. Their age varied between 34 and 70 years (mean 45). They described their psychiatric disabilities in terms of psychosis, bipolar disorder, compulsive disorders, chronic drug addiction, and comorbidity. All lived alone in different types of accommodations varying from villa to assisted housing. Five were singles with parents and siblings as next of kin. Four were divorced with between one and three children. All had at least compulsory school. Five had secondary school, and one had a university degree and some professional experience. All persons described their economic situation as difficult; their earnings were based on disability payments, retirement pensions, and social security allowance. One person had 50\% employ- ment and 50\% pension.

\subsection{Interviews}

Individual interviews were conducted according to Mishler [22] as everyday jointly constructed dialogues. Because of the vulnerability of the informants, extensive time was given to make the situation as comfortable as possible when the researchers presented the study and introduced the interview. An interview guide was used as a support and started with an overarching question: "Can you please tell me what SC really means to you?” The informants were encouraged to narrate more thoroughly about feelings, thoughts, and situations in relation to their contacts with the nurses at the SC. They were asked about how, when, and why they contacted the SC and what was then of significance to them. The first author (ACJ) conducted all interviews at the end of 2010 and the beginning of 2011. The interviews lasted between 40 and 85 minutes. The interviews were tape-recorded and transcribed verbatim. Ethical considerations related to the study followed the Swedish law for human research [23]. Permission to conduct the study was obtained from the head of the unit. All approached informants got an invitation and written and oral information, first from a nurse and second from the interviewer.

\subsection{Analysis}

The interpretation of the text consisted of naive reading, structural analysis, and a comprehensive understanding [19]. The naive reading process started with repeated readings of each interview and then all interviews, to give the researchers a sense of the meaning as a whole of the disabled person's lived experience of the 24-hour-aday support center. The naive understanding of the phenomenon was described in terms of what the text was sensed to be about, which were validated or invalidated by the structural analyses. The structural analysis started with efforts to identify and formulate themes, that is, threads of meaning. This process meant identifying meaning units in relation to the naive understanding. Thereafter, the meaning units were condensed and expressed in everyday words, grouped together by reflections on their similarities and differences. By further condensation and at times abstraction, the groups of condensed meaning units formed subthemes that were processed further to themes. Thereafter, the themes were reflected on in the light of the naive understanding for possible validation. As a whole, the sensed understanding of the meaning was validated. The naively sensed ideas became clear and clarified through the structural analysis. The comprehensive understanding was interpreted by summarizing the themes and subthemes, and reflecting on them in light of the meaning of the disabled person's lived experience of 
the 24-hour-a-day support center. The researchers' (ACJ, $\mathrm{IP}$, and $\mathrm{AB}$ ) pre-understanding has been formed by being registered psychiatric health nurses and RNTs with several years of professional experience. AB and ACJ also are experienced as researchers within the psychiatric health field.

\section{FINDINGS}

The naive reading revealed that the informants experienced the meaning of the SC in light of their past, present, and future life situations and psychiatric illnesses. They were rather content with their lives, especially in comparison to times before the start of the SC. Now they could handle their anxiety and loneliness in a secure way without having to turn to the hospital for help. They believed in improvements in their lives to come. The SC played a big role in this change in beliefs, which appeared as an increased sense of normality and hopes for a future normal life. It seemed as if the informants felt strengthened by the contacts with the nurses and the SC. Phoning, visiting, or staying overnight could make a positive change in their immediate states of mind, just as it could change the process of maintaining psychiatric health and preventing relapse. The SC also seemed to have a function as a comforting, welcoming, homelike place to go, to have people around, meet others in similar situation, greet friends, have a cup of coffee, and have some joy/fun.

The structural analysis revealed two themes in which theme 1) Becoming aware of myself as a person-had a deeper dimension; it concerned the informants' lived experiences of the meaning of the SC related to their inner self, while theme 2) Having a lifeline and belongingness-concerned the informants' lived experience of the SC in relation to an environmental dimension.

\subsection{Becoming Aware of Myself as a Person}

The text revealed this theme as having a deeper inner dimension concerning the informants' experienced meaning of the SC related to their inner self. The subthemes were: 1) feeling a positive change toward normality and 2) feeling free to make one's own decisions.

Subtheme: Feeling a positive change toward normality The informants looked filled with warmth when they were talking about how encouraging it felt to contact the SC and always feel welcomed. This was a new, positive sensation in comparison to former experiences in their lives of being rejected. To feel respectfully encountered and confirmed as a peer seemed to arouse feelings of strengthened value, self-esteem, and self-confidence. "There are moments when the invisible border/difference between the staff and us persons are dissolved, which 'feels"'... (I:2). Being at the SC was described as pro- viding an increased sense of peace and harmony. "It feels more human here... gives peace and serenity in my mind..." (I:5). The atmosphere, the encountering and everything else at the SC strengthened a feeling of being more normal than abnormal, and seemed to contribute to another way of looking at you. As feeling helped to see you with your own eyes, and not through others. Hope for a future normal life grew stronger.

Here, I do not feel like a patient, more as visiting any place in the city, more of a human atmosphere and not patient-staff relations (But, they have a privilege at the assisted housings here. Getting healthy food regularly, I eat, but not what I want to. I cannot afford it) (I:5).

Subtheme: Feeling free to make one's own decisions

The feeling of freedom in relation to the use of the SC was salient and was upheld as a pleasant experience that the informants wanted more of. Previous experiences of feeling restricted by others' demands came up in comparison. Having the possibility to decide if and when to phone, to visit, and to come and go as one pleased was highly appreciated. There was nobody trying to decide what is best for you. "...feeling freer, since not being pulled" (I:1). To have freedom to make contacts without having to make appointments was also a sort of freedom. This also meant the possibility to leave the SC in case of being uncomfortable. Such a situation was unusual but had happened when a visitor was distressed. "A visitor made me feel uncomfortable. It depends on their state... The hell with it... then you just go home” (I:4). In other words, the informants experienced freedom to use the SC as they desired and needed just as they also felt free to just be. They were free to make their own choices and that these choices were accepted and respected was restorative.

What SC means... it is that you can come here when and if you want to. Have a cup of coffee, talk, have fun or even stay over... (I:3). I do not need any therapeutic talk. I look after myself as I want. I have my friends here (I:5). SC means very much to me. You can come, sit down... yes whatsoever (I:4).

\subsection{Having a Lifeline and Belongingness}

The text revealed this theme that concerned experiences of what the SC meant to the informants in a more relational and environmental way. Just merely the existence of the SC and having the availability to go to a place like this meant a great deal. The SC meant support in several different ways. The informants might desire the harmony of the place itself or getting into a conversation with and feeling supported by the nurses. Other times they desired the support or company of other visitors and friends who were in a similar situation. The subthemes were 1) having security through available support and 2) having a comforting and welcoming place to go to. 
Subtheme: Having security through available support The feeling of having available supporting help 24 hours a day, whenever they were in need of it, made a big difference to the informants' sense of security. Knowing that there is always someplace to call and that someone answers and listens was a part of this sense of security, as was the possibility of visiting and even staying overnight when it felt as if everything could collapse. Feelings of loneliness and anxiety can be experienced as almost unbearable at times. The informants gratefully expressed how the staff attempted to prevent relapse and hospitalization at difficult times. As expressed by informants:

If it was not for the SC I would not be alive today. They have saved my life" (I:9). The SC means very much to me... they have supported me much when I was feeling pretty damn bad, they saw my bad shape here, and then even came to my home (I:4). It has happened that I have come here in the middle of the night and slept over -thunderstorms scare me (I:7).

Such efforts made a difference because all the informants had experienced previous rejection when they sought help at the Psychiatric Emergency Department (PED) in the larger city. All expressed painful memories of feeling psychiatrically ill, trying to avoid having to travel to the PED, eventually having to give up because of anxiety and psychiatric illness, seeking help at PED and then being dismissed as not being sick enough, and left to go home. This was experienced as degrading. They now felt relieved since not having to experience such disparaging encounters again. Further, informants described the meaning of the SC:

At times I need a conversation with someone. Since I know how it starts. Do not sleep at nights. Become incredibly quick-witted, so seductive... you are drawn with it... And then it turns, you become totally crazy. You crack... and all that you have built up. So I need someone to turn to, that can take care of me and say... aaaaa, take it easy now and slow down (I:2). I have had all support I needed at daytime, but not at nights (I:8).

Subtheme: Having a welcoming, homelike place to go to. The SC appeared to work as a stabilizing factor in the lives of the informants. "I do not have many friends, but I have SC to go to whenever I feel like it” (I:4). The nurses were experienced as always welcoming, and they acknowledged the informants' experiences in a respectful manner no matter what they were about. It also seemed as if some support came from getting just enough structure (balance) in their daily lives. Some often visited the SC as part of their daily routine. Some were sensitive to too much structure because they felt that they were too much controlled. At the SC they felt at home; they could relax-Here it feels like an oasis of... just being (I:1) — and get a sense of togetherness and meaning. To meet others in similar situation could be a relief and to meet friends was a joy. Such joy could also mean having a cup of coffee, watching TV together, having fun and playing games. Here I can play games and have fun afternoons and evenings, sit down on a sofa, have a cup of tea, watch the large flat-screen TV. My own TV broke down this summer (six months ago) (I:5). Or at times the SC gave feelings of ease and comfort in just having people around. Human warmth alleviated feelings of being alone as well as loneliness.

Specific with The SC is that everyone is welcome always. To meet staff with time to have a conversation actually means-warmth. In loneliness you are drawn here (I:6). I have made a schedule, at first I take the bus to the meeting point open at daytime. We chat and play games until two a clock pm. Then I go home for dinner. Thereafter I go to the SC at six pm. I had a job that I had to leave... I got sick. I miss not having a job to go to (I:4).

\section{COMPREHENSIVE UNDERSTANDING AND REFLECTION}

The lived experience among persons with psychiatric disabilities of a newly established 24-hour-a-day community support center was interpreted through summarizing and reflecting on the research question, the naive reading, the sub-themes and themes, and the context of the study, all meaning Making me feel almost like an ordinary person. This overarching meaning incorporates the person's past with his/her present together with a direction for the future and a hope for the future of a more fulfilled life. The meaning of the SC as a whole can be understood as promoting the recovery processes by strengthening the person's inner development processes of self together with providing a supportive outer environment. Recent research [24] describe recovery similarly_as a fundamental personal process involving an inner new sense of self and hope, which requires outer conditions that facilitate a positive environment of healing. To be even more specific, Slade and colleagues [25] emphasize that recovery processes comprise connectedness, hope and optimism about the future, identity, meaning in life, and empowerment. These are aspects that can be recognized as what SC support means to psychiatrically disabled people: inner and outer support that can promote healthy transition processes to a sense of ordinariness.

Having a lifeline and belongingness illuminate the meaning of SC as an organized service that enhanced the psychiatrically disabled's individual processes toward ordinariness and normality. The SC environment meant a sense of belongingness by providing a welcoming, homelike atmosphere that offered warmth and harmony. Schröder, and colleagues [26] emphasized the qualities 
of the physical environment as aesthetically pleasing and providing a pleasant, homelike atmosphere for psychiatric patients. Grönberg Eskel [27] found the environment or situation as decisive for the psychiatrically disabled person's chance to feel like others. Some environments frame a person as psychiatrically disabled, and others do not.

Environments like the SC may enhance feelings of being like anyone else. An organizational aspect may play a role in this: the SC's lack of focus on diagnosis and medical treatment. That SC was non-medical, and because the persons were without documentation, they were treated without "diagnoses" and medication. This can make the SC empowering to persons - to how they experience and view themselves. The meaning of such treatment could be understood by contrasting it to a person's being seen only as a diagnosis and not as a unique human being. Illich ([28], p. 96) argued that "diagnosis” gives the person a certain role that he/she associates with incapacity, uncertainty, and non-recovery and separates him/her from the normal and healthy. Goffman [29] described this as a role-deprivation process, degrading the person's self-perception by peeling off much of what the person has perceived as self-a sort of objectification process. Today some may view this description as anachronistic, but research suggests otherwise. For instance, Lilja and Hellzén [30] showed how former patients experienced hospitalization in psychiatric care as "being seen as a disease" - in other words as a diagnosis; in contrast, care as a "light in the darkness" could occur when patients experienced being treated as normal human beings. When the persons in our study spoke about the SC, they always compared it to former experiences. In these comparisons, the SC meant support in terms of a physical place organized as a constant lifeline. When someone was having difficult times, the around-the-clock availability together with always being welcomed was experienced as unique-like at night-times, when loneliness, anxiety, and worry beset the person. Then the SC meant a lifeline that gave relief and security when things appeared to collapse. A contact or stay overnight at the SC could be a saving grace. Also Borg and Kristiansen [31] found the availability of helpers at difficult times, such as when hearing voices or experiencing extreme anxiety, was particularly helpful to persons with psychiatric illnesses. Without available helpers at places like, for instance, the SC, psychiatric hospital care becomes the only alternative and involves care that seldom provides an adequate healing environment. The SC was established as a result of "persons" own expressed needs for support organized with around-the-clock availability. Their experience of this kind of support as so helpful points to the importance of communities that take the responsibility to provide such a "first aid kit" with em- phasis on the 24-hour availability, a welcoming attitude, and a focus on the person's here-and-now situation and daily living.

In the theme of Becoming aware of myself as a person, the SC appeared to strengthen the processes within the person's inner self as a growing awareness of the inner world in comparison to the outer world. Such awareness emerges in other studies as a dimension in positive development processes. Lindell [32] found that psychiatric patients viewed themselves as more aware of their inner self when not feeling ill and hospitalized. The awareness in our study perhaps meant increased awareness of positive changes within one's inner self, of feelings and thoughts that raise the hope of becoming ordinary. This ordinariness emerged as something very positive and desired. To ordinary people, this may sound strange, because they often wish to be special and deviate from the ordinary crowd. However, the positive dimension of the comprehensive understanding of "feeling almost ordinary" may be best understood in comparison to what it means to be in the world with a long-term psychotic illness, as shown by Syrén [33]. To be psychotic is to be someone other than an ordinary person. The disease represented a peculiarity that showed itself by different behavior and lack of feelings that ordinary people have. Persons with long-term psychotic illness live in a borderland between the usual and unusual. They struggle with doubts about being healthy or ill and about being usual or unusual. In the study by Lindell [32], psychiatric users viewed ordinary persons as persons with abilities, who could feel good at home, cook, work, travel, go by bus, and move around freely in the community. This probably seems rather unpretentious and taken-for-granted by ordinary people. But to people with psychiatric disabilities, this image may evoke the hope of increased well-being, abilities, autonomy, and independence. This enhances the meaning of the theme of feeling free to make one's own decisions-a freedom that emerged as something highly valued. It actually seemed as if the awareness of the freedom to make decisions had a sense of novelty and was restorative to the person's inner self.

Another dimension of the increased awareness of one's inner self is related to what it meant to be together and to interact with nurses and others at the SC. Being treated with respect as an equal fellow or peer strengthened feelings of being more ordinary rather than deviant. The permissive relational climate offered meant a freedom to relate on one's own terms. This created preconditions for building relationships, cautiously, at his/her own pace. Here there was all the time needed to practice relating to others in order to rebuild one's own inner self. Thus, being together with the nurses and others was of great importance but not always in terms of close relationships. Rather, closeness could be avoided probably 
as a strategy to survive in existential meaning because of painful experiences earlier in life (cf. [34-36]). Sandin [35] holds that a person who suffers from schizophrenia could withdraw from life and development in an attempt to get away from destructive anxiety and thereby lose the communication inherent in a relation, which needs to be able to develop. Thus, human relations are of crucial importance for the ability of a person to develop his/her own confident self. Martin Buber [37] said, "The Self is created in the meeting with Thou". From an existential perspective, the SC seemed to have what is required to be a genuine meeting place so that around the psychiatrically disabled persons there was a group of staff who work continuously and reliably, hopefully for many years, in enduring everyday contacts.

Seen this way, SC nurses and others gently supported the development of self and identity. That signals from others contribute to creating the individual's self is in accordance with the symbolic interactionism; self and one's identity are created by a constant interaction process with the environment [38]. Ricoeur [39] viewed "others" as very important in order to understand the self, because such understanding is impossible without the other, and vice versa. He believed that an individual's identity has a sense of stability but can engage in self-creation. In other words, people can have both a sense of grounding and yet also experience changes to the self. Slade [40] argues that to promote recovery processes, it is vital to develop a positive identity in comparison to one that is illnessdefined. This resembles the work of Davidson and Strauss [41], who claim that rediscovering and reconstructing a dynamic sense of self is vital in the recovery process. Kogstad and colleagues [24] found that in mental health persons, the recovery process of "rediscovering oneself" concerned relationships with psychiatric professionals and others. Positive encounters enhanced new feelings of dignity and self-respect. In our study, the disabled persons were encountered in a positive way; conversations and having a talk were much appreciated. This seemed to strengthen feelings of equality and normality. Simple things like ordinary conversations and "talk" about common things instead of disease and related problems make psychiatrically disabled persons feel recognized as ordinary human beings $[31,42,43]$. Thus, for a person to have ordinary conversations and talks with nurses and others appears as healing a person's self by underpinning selfconfidence and a sense of ordinariness.

\section{Methodological Considerations}

To ensure trustworthiness of the research findings, steps were taken along the research process in accordance with criteria for credibility, dependability, and transferability [44]. Efforts to strengthen the credibility were made by striving for researcher credibility [45], ensuring that ca- pable persons, with professional competence and awareness of the sensitivity of the study, were conducting the study. Testing the interview guide was also such an endeavor. Most participants spoke openly and richly in the interviews, while one single participant had more limited language, likely influenced by neuroleptic medication. However, altogether the interviews generated rich data to the research focus. By the ninth interview, similar topics were recognized as repeated and saturation was considered to be reached. All interviews were conducted by ACJ. In order to strengthen the dependability a description of the analysis process was made to facilitate for readers to follow development of the themes. Citations from the interviews were also presented to validate that the themes represented the informants' lived experience of the SC. All three authors participated in all steps of the analysis process, reviewed the texts independently, and discussed all codes back and forth and reached a consensus on the themes and thereafter the comprehensive understanding. To strengthen the credibility of the study as a whole, descriptions of the steps of the research process were made. Through this procedure readers should be able to judge the transferability of the findings. Limitations and threats to credibility included the researcher's decision to let the staff select the informants. There is a risk that the staff selected only positive informants, while the circumstances suggested their judgment was trustworthy. In consideration of ethical aspects and the vulnerability of the group, this selection was considered the best.

\section{ACKNOWLEDGEMENTS}

The authors warmly thank the persons who took part in the interviews. Thanks also to The Research Platform in Collaboration for Health, Kristianstad University and The Research Board Kristianstad University for funding.

\section{REFERENCES}

[1] Funk, M., Drew, N., Freeman, M., Faydi and Edwige, V. (2010) Mental Health and development: Targeting people with mental health conditions as a vulnerable group. World Health Organisation.

[2] NBHW (2013) National evaluation in 2013-Care and interventions for depression, anxiety and Schizofrenia. Recommendations, assessments and summary. National Board of Health and Welfare, Stockholm.

[3] NBHW (2011) National Guidelines for Psychosocial Interventions in Schizofrenia or Shitzofrenia-Type conditions-Support for Guvernance and Management. National Board of Health and Welfare, Stockholm.

[4] Janlöv, A.C. and Berg, A. (2013) The nurses' voice of working in a newly established community based 24-hour support center for people with psychiatric disabilities. Open Journal of Nursing, 3, 195-201. 
http://dx.doi.org/10.4236/ojn.2013.32026

[5] Rosenberg, D. (2009) Psychiatric disability in the community. Surveying the social landscape in the post-deinstitutional era. Ph.D. Thesis, Umeå University, Umeå.

[6] Perry, Y., Henry, J.D., Sethi, N. and Grisham, J.R. (2011) The pain persists: How social exclusion affects individuals with schizophrenia. British Journal of Clinical Psychology, 50, 339-349.

http://dx.doi.org/10.1348/014466510X523490

[7] Lundberg, B. (2007) Sociodemographic and clinical factors related to devaluation/discrimination and rejection experiences among persons of psychiatric health services. Social Psychiatry and Psychiatric Epidemiology, 42, 295300. http://dx.doi.org/10.1007/s00127-007-0160-9

[8] Van Zelst, C. (2009) Stigmatization as an environpsychiatric risk in schizophrenia. Schitzofrenia Bullentin, 35, 293-296.

[9] Lindqvist, R., Markström, U. and Rosenberg, D. (2010) Psychiatric disability in the society: Actors, service, reforms. Gleerups förlag, Lund.

[10] Nyström (1999) Serious mentally disturbed people's everyday lives. Ph.D. Thesis, Gothenburg University, Gothenburg.

[11] Erdner, A., Andersson, L., Magnusson, A. and Lutzén, K. (2009) Varying views of life among people with longterm mental illness. Journal of Psychiatric and Mental Health Nursing, 16, 54-60. http://dx.doi.org/10.1111/jpm.2009.16.issue-1

[12] Erdner, A., Nyström, M., Lutzén, K. and Severinsson, E. (2002) Psycho social disadvantages in the lives of persons with long term psychiatric illness in a Swedish community. Journal of Psychiatric and Psychiatric Health Nursing, 9, 457-463.

[13] Klamas, M. (2010) By own power along with others. Persons with psychiatric disabilities, social support and recovery. Ph.D. Thesis, Gothenburg University, Göteborg.

[14] SFS (1982) Health and Medical Services Act. Ministry of Health and Social Affairs, Stockholm.

[15] SFS (2001) Social Services Act. Ministry of Health and Social Affairs, Stockholm

[16] SFS (1993) Act of support and service for the disabled. Ministry of Health and Social Affairs Stockholm.

[17] NBHW (2012) National supervision of municipal service to persons with psychiatric disabilities 2009-2011. National Board of Health and Welfare, Stockholm.

[18] Thornicroft, G., Rose, D., Huxley, P., Dale, G. and Wykes, T. (2002) Editorial: What are the research priorities of mental health service persons? Journal of Mental Health, 11, 1-5. http://dx.doi.org/10.1080/096382301200041416

[19] Lindseth, A. and Norberg, A. (2004) A Phenomenological hermeneutical method for researching lived experience. Scandinavian Journal of Caring Sciences, 18, 145153. http://dx.doi.org/10.1111/j.1471-6712.2004.00258.x

[20] Ricoeur, P. (1976) Interpretation Theory. Discourse and the Surplus of Meaning. Christian University Press, Fort Worth, Texas.
[21] Ricoeur, P. (1992) From Text to Action. An Anthology on Hermeneutics. Symposion, Stockholm.

[22] Mishler, E.G. (1986) Research Interviewing: Context and Narrative. Harvard University Press, London.

[23] Codex (2012) Rules and guidelines.

[24] Kogstad, Ekeland and Hummelvoll (2011) In defence of a humanistic approach to mental health care: Recovery processes investigated with the help of persons' narratives on turning points and processes of gradual change. Journal of Psychiatric and Mental Health Nursing, 18, 479-486. http://dx.doi.org/10.1111/j.1365-2850.2011.01695.x

[25] Slade, M., Williams, J., Bird, V., Leamy, M. and Le Boutillier, C. (2012) Recovery grows up. Journal of Mental Health, 21, 99-104. http://dx.doi.org/10.3109/09638230903191256

[26] Schröder, A., Ahlström, G. and Wilde, B.W. (2006) Patients' perceptions of the concept of the quality of care in the psychiatric setting: A phenomenographic study. Journal of Clinical Nursing, 15, 93-102.

[27] Grönberg Eskel, M. (2012) From closed institutions to institutionalized care. Ph.D. Thesis, Karlstad University, Karlstad.

[28] Illich, I. (1975) Limits to medicine. Boyars Publishers, London.

[29] Goffman, E. (1991) Asylums. Essays on the Social situation of Mental Patients and other inmates. Penguin Books Ltd.

[30] Lilja, L. and Hellzén, O. (2008) Former patients' experience of psychiatric care: A Qualitative investigation. International Journal of Mental Health Nursing, 17, 279286. http://dx.doi.org/10.1111/j.1447-0349.2008.00544.x

[31] Borg, M. and Kristiansen, K. (2004) Recovery-oriented professionals: Helping relationships in mental health services. Journal of Mental Health, 13, 493-505. http://dx.doi.org/10.1080/09638230400006809

[32] Lindell, L. (2003). Between healthy and ill: A study of Psychiatric Outpatient Care. Ph.D., Thesis, Lund University, Lund.

[33] Syrén, S. (2010) The unsaid and the unspoken suffering. Life for people with chronic psychotic illness and their families. Ph. D Thesis, Linnéuniversitetet, Kalmar, Växjö.

[34] Laing, R.D. (2010) The divided self. An Existential Study in Sanity and Madness. Penguin Classics.

[35] Sandin, B. (1986) The zebra stripe poodle core. Rabén \& Sjögren, Stockholm.

[36] Belin, S. (1987) Schizofrenia treatment. Psychiatry of life and death. Natur och Kultur, Stockholm.

[37] Buber, M. (1971) I and Thou. Simon and Schuster, New York.

[38] Blumer, H. (1969) Symbolic interactionism. Prentice Hall inc, New Jersey.

[39] Ricoeur, P. (1992b) Oneself as another. The University of Chicago Press, Chicago.

[40] Slade, M. (2009) Editorial. The contribution of mental health services to recovery. Journal of Mental Health, 18, 
376-371. http://dx.doi.org/10.3109/09638230903191256

[41] Davidson, L. and Strauss, J. (2011) Sense of self in recovery from severe mental illness. British Journal of Medical Psychology, 65, 131-145.

http://dx.doi.org/10.1111/j.2044-8341.1992.tb01693.x

[42] Repper, J. (2000) Adjusting the focus of mental health nursing: Incorporating service persons' experiences of recovery. Journal of Mental Health, 9, 575-587.
[43] Williams, C.C. and Tufford, L. (2012) Professional competencies for promoting recovery in mental illness. Psychiatry, 75, 190-201.

http://dx.doi.org/10.1521/psyc.2012.75.2.190

[44] Lincoln, Y.S. and Guba, E. G. (1985) Naturalistic Inquiry. Sage Publications, London.

[45] Patton, M.Q. (2002) Qualitative Research \& Evaluation Methods. 3rd Edition, Sage Publications, Thousand Oaks. 